\title{
SAFE MULTI-CONTROLLER ADAPTIVE SWITCHING USING CLOSED-LOOP DATA*
}

\author{
ARVIN DEHGHANI ${ }^{\dagger}$
}

\begin{abstract}
Consider an interconnection of an unknown or partially known plant and a known stabilizing controller, and assume that some knowledge of the closed-loop system is available. Suppose, on the basis of that knowledge, the use of a new controller appears attractive. We present analysis and novel tests using a limited amount of experimental and possibly noisy data obtained from the existing closed-loop for verifying robust stability and performance with the new controller before it is switched in. The importance of this capability arises in multi-controller adaptive switching including iterative identification and control algorithms and multiple-model adaptive control. The results cover the linear and nonlinear cases.
\end{abstract}

Key words: Multiple Model Adaptive Control, Multi-Controller Switching, Iterative Identification and Control, Robust Control, Nonlinear Control, Adaptive Control

1. Introduction. During the operation of a stable closed-loop system - consisting of a plant and a pre-designed stabilizing controller in a standard feedback setting - situations arise where the controller needs to be partially or entirely replaced by a newly designed controller. Such scenarios include the situation where the plant requires the use of a new controller due to ageing or damage, or where one seeks to improve some aspects of the closed-loop performance, e.g. introducing a nonlinear controller in place of a linear controller connected to a linear plant to secure faster rise-time without increasing the percentage overshoot [21].

Many multi-controller adaptive switching algorithms do not explicitly rule out the possibility of placing a destabilising controller in the closed-loop $[1,7,10,15,16]$. Even if the new controller is ensured to be stabilising, performance verification with the new controller to satisfy the performance requirements and guarantee a better performing closed-loop is not straightforward.

In adaptive control, it is very frequently the case that a model is explicitly or implicitly constructed, and the performance of the model is compared with the actual system. When the actual system comprises a plant in combination with a controller, the model may for example comprise an estimate of the plant obtained by identification in combination with the same controller. Or the model may be a desired closed-loop transfer function, and the actual system comprises the closed loop formed by the actual plant and the controller. Thus a measure of quality for the model is usually an a priori agreement. The central difficulty is that a model may be a good

*Dedicated to Brian Anderson on the occasion of his 70th birthday. This work was supported by the ARC Discovery-Projects Grant DP1095290.

$\dagger$ A. Dehghani is with the Centre for Neural Engineering, Melbourne School of Engineering, The University of Melbourne, Parkville VIC 3010 Australia. E-mail: arvin.dehghani@unimelb.edu.au 
model with one set of experimental conditions, but not with another set.

We shall review techniques for verifying the closed-loop stability [9], and its extension for treating robust performance verification $[5,6]$ with the introduction of a new linear controller $C_{1}$ using a limited amount of noisy input-output experimental data obtained from the stable closed-loop $\left[P, C_{0}\right]$. We shall also present analysis results that are general enough to cover nonlinear plant and controllers [2-4] and lay the foundation for extending the aforementioned results in the linear case. In particular, we extend the applicability of the 'kernel representation' of a nonlinear system as a generalization for the existing results in the linear case.

The structure of the paper is as follows. Section 2 collects the required definitions and notations from the relevant literature. We shall state the problem of interest in Section 3 before presenting the stability verification tests in Section 4 and performance verification tools in Section 5 in a linear setting. Section 6 present the analysis results for the extension of the results of the preceding sections to the nonlinear case. Section 8 contains concluding remarks and future research directions.

2. Preliminaries. The notations are standard and borrowed from $[20,22]$ in the linear case and $[2,4,11,18]$ in the nonlinear case.

We shall denote by $\mathscr{H}_{\infty}$ the space of functions bounded and analytic in the open right-half complex plane, and the same function spaces with prefix $\mathscr{R}$ their realrational proper subspaces. The eigenvalues of $A \in \mathbb{C}^{m \times m}$ are denoted by $\lambda_{1}, \cdots, \lambda_{m}$ and its spectral radius $\rho(A)=\max _{1 \leq i \leq m}\left|\lambda_{i}\right|$. The determinant of a matrix is denoted by det and its singular values by $\sigma_{i}(\cdot)$ with the largest singular value $\bar{\sigma}(\cdot)$ and the smallest singular value $\underline{\sigma}(\cdot)$. The number wno $(\cdot)$ denotes the winding number of the Nyquist diagram of a scalar transfer function, evaluated on a contour along the imaginary axis and indented to the right around any pure imaginary pole. The nearest integer function nint [.] returns the integer closest to [.] with the additional rule that half-integers are always rounded to even numbers. We utilize the coprime stable factor representations of the plant $P$ and the controllr $C$, and assume that the plant and all controller transfer functions are always proper. We denote $G(j \omega)^{*}$ as the complex conjugate transpose of frequency-response function $G(j \omega)$ at each $\omega$, i.e. $G(j \omega)^{*}=G(-j \omega)^{T}$.

Let $\mathscr{L}_{2}^{m}[0, \infty)$ (in short $\mathscr{L}_{2}^{m}$ or $\mathscr{L}_{2}$ ) denote a vector space of $\mathbb{R}^{m}$ valued square integrable functions with norm defined by $\|f\|:=\left(\int_{0}^{\infty} f^{T} f d t\right)^{1 / 2}$. Define a truncation operator, $\mathcal{T}_{T}$, on the vector space of functions mapping from $\mathbb{R}$ to $\mathbb{R}^{m}$ by $\mathcal{T}_{T} f(t)=f(t)$ if $t \leq T, \mathcal{T}_{T} f(t)=0$ if $t>T$. Let $\mathscr{L}_{2 e}^{m}[0, \infty)$ (in short $\mathscr{L}_{2 e}^{m}$ or $\mathscr{L}_{2 e}$ ) denote the extended space of functions $f: \mathbb{R}^{+} \rightarrow \mathbb{R}^{m}$ satisfying $\mathcal{T}_{T} f \in \mathscr{L}_{2}, \forall T>0$. Analogously, let $\mathscr{H}_{2}$ denote a vector space of matrix-valued functions $F(s)$ analytic in the open righthalf plane such that $\|F\|:=\sup _{\sigma>0}\left(\frac{1}{2 \pi} \int_{-\infty}^{\infty}|F(\sigma+j \omega)|^{2} d \omega\right)^{1 / 2}<\infty$. Also, let $\mathscr{H}_{\infty}$ denote the space of bounded functions in the open right-half plane such that 
$\|F\|_{\infty}:=\sup _{\omega \in \mathbb{R}} \bar{\sigma}[F(j \omega)]<\infty$, where $\bar{\sigma}(F)$ denotes the largest singular value of $F(s)$. We shall denote the real rational subspace of $\mathscr{H}_{2}$ (resp. $\mathscr{H}_{\infty}$ ) by $\mathscr{R}_{2} \mathscr{H}_{2}$ (resp. $\mathscr{R}_{\infty}$ ). Since $\mathscr{L}_{2}$ and $\mathscr{H}_{2}$ form an isomorphism through the Laplace transform (the Parseval's relations), $f(t) \in \mathscr{L}_{2}$ and $F(s) \in \mathscr{H}_{2}$ will be used interchangeably.

DEFINITION 2.1. The unwrapped phase of a scalar transfer function is denoted by unwarg and refers to the phase of the frequency response when it is in the form of a continuous function of the frequency. The unwrapped phase is computed from the phase frequency response by changing absolute jumps greater than $\pi$ to their $2 \pi$ complements, and ensures that all appropriate multiples of $2 \pi$ are included.

Consider the interconnection $[P, C]$ in Fig. 2.1.

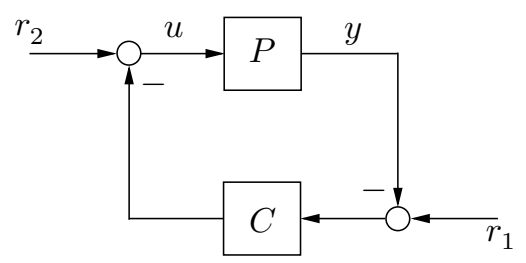

Fig. 2.1. A Standard Feedback Configuration

Definition 2.2. The interconnection $[P, C]$ is "well-posed" if the transfer function matrix mapping $\left[\begin{array}{l}r_{1} \\ r_{2}\end{array}\right]$ to $\left[\begin{array}{l}y \\ u\end{array}\right]$ exists. Put another way, $[P, C]$ is well-posed if $(I-C P)^{-1} \in \mathscr{R}$.

Given such well-posedness in Fig. 2.1 we have

$$
\left[\begin{array}{l}
y \\
u
\end{array}\right]=\left[\begin{array}{c}
P \\
I
\end{array}\right](I-C P)^{-1}\left[\begin{array}{ll}
-C & I
\end{array}\right]\left[\begin{array}{l}
r_{1} \\
r_{2}
\end{array}\right]=H_{[P, C]}\left[\begin{array}{l}
r_{1} \\
r_{2}
\end{array}\right] .
$$

Definition 2.3. The interconnection $[P, C]$ is said to be "internally stable" if it is well-posed and $H_{[P, C]} \in \mathscr{R} \mathscr{H}_{\infty}$.

Definition 2.4. The ordered pair $\{N, M\}$, with $M, N \in \mathscr{R}_{\infty}$, is a rightcoprime factorization (rcf) of $P \in \mathscr{R}$ if $M$ is invertible in $\mathscr{R}, P=N M^{-1}$, and $N$ and $M$ are right-coprime over $\mathscr{R} \mathscr{H}_{\infty}$. Furthermore, $\{N, M\}$ is a normalized $\mathrm{rcf}$ of $P$ if $\{N, M\}$ is a $\operatorname{rcf}$ of $P$ and $M^{*} M+N^{*} N=I$.

Definition 2.5. The ordered pair $\{\tilde{U}, \tilde{V}\}$, with $\tilde{U}, \tilde{V} \in \mathscr{R} \mathscr{H}_{\infty}$, is a left-coprime factorization (lcf) of $C \in \mathscr{R}$ if $\tilde{V}$ is invertible in $\mathscr{R}, C=\tilde{V}^{-1} \tilde{U}$, and $\tilde{U}$ and $\tilde{V}$ are left-coprime over $\mathscr{R} \mathscr{H}_{\infty}$. Furthermore, $\{\tilde{U}, \tilde{V}\}$ is a normalized lcf of $C$ if $\{\tilde{U}, \tilde{V}\}$ is a lcf and $\tilde{V} \tilde{V}^{*}+\tilde{U} \tilde{U}^{*}=I$.

In the sequel, the use of 'normalized' coprime factors may become necessary at places.

Definition 2.6. The graph symbol of $P, G$, and the inverse graph symbol of $C$, 
$\tilde{K}$, are defined by

$$
G:=\left[\begin{array}{l}
N \\
M
\end{array}\right] \quad, \quad \tilde{K}:=\left[\begin{array}{ll}
-\tilde{U} & \tilde{V}
\end{array}\right]
$$

Theorem 2.7. [20, Prop. 1.9] Let $G$ and $\tilde{K}$ be defined as in (2.1). Then the following are equivalent:

i. $[P, C]$ is internally stable;

ii. $(\tilde{K} G)^{-1} \in \mathscr{R} \mathscr{H}_{\infty}$;

iii. $\operatorname{det}(\tilde{K} G)(j \omega) \neq 0 \forall \omega$ and $\operatorname{wno} \operatorname{det}(\tilde{K} G)=0$.

DEFINITION 2.8. For a (not necessarily scalar) $P$, the "robust stability margin" $b_{[P, C]}$ is defined by

$$
b_{[P, C]}:= \begin{cases}\left\|H_{[P, C]}\right\|_{\infty}^{-1}, & \text { if }[P, C] \text { is internally stable } \\ 0 & \text { otherwise }\end{cases}
$$

Clearly, larger $b_{[P, C]}$ corresponds to the smaller norm, but this norm cannot be smaller than unity, which means that for any $P$ and $C, b_{[P, C]} \in[0,1]$; see [20].

Definition 2.9. [20, p. 38] The "point-wise version" of $b_{[P, C]}$ is defined by

$$
\rho(P, C, \omega)=1 / \bar{\sigma}\left(\left[\begin{array}{l}
P \\
I
\end{array}\right](I-C P)^{-1}\left[\begin{array}{ll}
-C & I
\end{array}\right]\right)(j \omega)
$$

Definition 2.10. For stable $[P, C], b_{[P, C]}=\inf _{\omega} \rho(P, C, \omega)$.

Note 2.11. Since $H_{[P, C]}=G(\tilde{K} G)^{-1} \tilde{K}$, one obtains

$$
\begin{aligned}
\rho(P, C, \omega) & =1 / \bar{\sigma}\left[\left(G(\tilde{K} G)^{-1} \tilde{K}\right)(j \omega)\right] \\
& =1 / \bar{\sigma}\left[\left((\tilde{K} G)^{-1}\right)(j \omega)\right]=\underline{\sigma}[(\tilde{K} G)(j \omega)]
\end{aligned}
$$

and if $[P, C]$ is stable $b_{[P, C]}=1 /\left\|(\tilde{K} G)^{-1}\right\|_{\infty}$.

TheOrem 2.12. [20] Let $P$ be scalar, and the gain margin and phase margin be denoted by $G M$ and $P M$, respectively. If $[P, C] \in \mathscr{R} \mathscr{H}_{\infty}$,

$$
G M \geq \frac{\left(1+b_{[P, C]}\right)}{\left(1-b_{[P, C]}\right)} \text { and } P M \geq 2 \arcsin \left(b_{[P, C]}\right) .
$$

2.1. Operator spaces. Consider an operator $\Sigma^{x}: \mathscr{L}_{2 e}^{m} \rightarrow \mathscr{L}_{2 e}^{k}$ with an initial condition $x \in \mathcal{X}_{\Sigma} \subset \mathbb{R}^{n}$.

Definition 2.13 (Causal). The operator $\Sigma^{x}$ is said to be causal if $\Sigma^{x}(f) \in \mathscr{L}_{2 e}^{k}$ is uniquely determined $\forall f \in \mathscr{L}_{2 e}^{m}$ and $\forall x \in \mathcal{X}_{\Sigma}$, and $\mathcal{T}_{T} \Sigma^{x} \mathcal{T}_{T}=\mathcal{T}_{T} \Sigma^{x}$ holds $\forall T>0$ and $\forall x \in \mathcal{X}_{\Sigma}$.

DeFINITION 2.14 (Causally Invertible). The operator $\Sigma^{x}$ is said to be causally invertible if it is causal, $m \equiv k$ holds and there exists a causal operator $\left(\Sigma^{x}\right)^{-1}$ : 
$\mathscr{L}_{2 e}^{m} \rightarrow \mathscr{L}_{2 e}^{m}$ (also denoted as $\left(\Sigma^{-1}\right)^{x}$ or $\left.\Sigma^{-1}\right)$ such that $\Sigma^{x}\left(\Sigma^{x}\right)^{-1}=\left(\Sigma^{x}\right)^{-1} \Sigma^{x}=I$, $\forall x \in \mathcal{X}_{\Sigma}$ holds, where $I$ denotes the identity operator.

DEFINITION 2.15 (Bounded). The operator $\Sigma^{x}$ is said to be bounded if it is causal and there exists a finite constant $\gamma$ and a scalar non-negative function $\phi$ with $\phi(0)=0$ such that $\left\|\Sigma^{x}(u)\right\|_{p} \leq \gamma\|u\|_{p}+\phi(x), \forall u \in \mathscr{L}_{2}^{m}, \forall x \in \mathcal{X}_{\Sigma}$. The minimum value of $\gamma$ that satisfies this inequality is called the gain and denoted by $\|\Sigma\|_{p_{i}}$.

DEFinition 2.16 (Weakly Lipschitz). The operator $\Sigma^{x}$ is said to be weakly Lipschitz (or weakly Lipschitz continuous) if it is causal and its Lipschitz semi-norm

$$
\left\|\mathcal{T}_{T} \Sigma^{x}\right\|_{L}:=\sup _{\substack{u, \nu \in \mathscr{L}_{2 e}^{m} \\ \mathcal{T}_{T} u \neq \mathcal{T}_{T} \nu}} \frac{\left\|\mathcal{T}_{T} \Sigma^{x} u-\mathcal{T}_{T} \Sigma^{x} \nu\right\|}{\left\|\mathcal{T}_{T} u-\mathcal{T}_{T} \nu\right\|}
$$

is finite for every $T>0$ and $x \in \mathcal{X}_{\Sigma}$.

DEFINITION 2.17 (Smoothing). The operator $\Sigma^{x}$ is said to be smoothing if it is weakly Lipschitz and for every $T>0, \gamma>0$ and $x \in \mathcal{X}_{\Sigma}$ there exists $t_{1}=t_{1}(T, \gamma, x) \in$ $(0, T)$ such that $\left\|\mathcal{T}_{t+t_{1}}\left(\Sigma^{x} \mathcal{T}_{t+t_{1}}-\Sigma^{x} \mathcal{T}_{t}\right)\right\|_{L} \leq \gamma$ holds for $\forall t \in\left[0, T-t_{1}\right]$.

Note 2.18. [19] Some interesting results are:

$i$. The sum (or cascade) of two weakly Lipschitz (resp. smoothing) operators is also weakly Lipschitz (resp. smoothing);

ii. $A B$ is smoothing if $A$ is smoothing and $B$ is weakly Lipschitz; $B A$ however is not necessarily smoothing;

iii. $[A, B]$ is well-posed if $A$ is smoothing and $B$ is weakly Lipschitz.

Definition 2.19 (Parameterized). The operator $\Sigma_{w}^{x}: \mathscr{L}_{2 e}^{m} \Rightarrow \mathscr{L}_{2 e}^{k}$ is said to be parameterized with $w \in \mathscr{L}_{2 e}^{l}$ if there exists an associated operator $\Sigma^{x}: \mathscr{L}_{2 e}^{l} \times \mathscr{L}_{2 e}^{m} \Rightarrow$ $\mathscr{L}_{2 e}^{k}$ such that $\Sigma_{w}^{x}(u)=\Sigma^{x}(w, u), \forall u \in \mathscr{L}_{2 e}^{m}, \forall w \in \mathscr{L}_{2 e}^{l}, \forall x \in \mathcal{X}_{\Sigma_{w}}$.

Definition 2.20 (Parametrically Linearly Bounded). A parameterized operator $\Sigma_{w}^{x}$ is said to be parametrically linearly bounded if there exists a finite constant $\gamma$ and a scalar function $\phi$ with $\phi(0)=0$ such that $\left\|\Sigma_{w}^{x}(u)\right\| \leq \gamma\left\|\left(\begin{array}{l}w \\ u\end{array}\right)\right\|+\phi(x) \forall u \in \mathscr{L}_{2 e}^{m}, \forall w \in$ $\mathscr{L}_{2 e}^{l}, \forall x \in \mathcal{X}_{\Sigma}$. The minimum value of $\gamma$ is called the parametric gain and denoted by $\left\|\Sigma_{w}\right\|_{p_{i}}$.

3. Problem Statement. Suppose the feedback control interconnection $\left[P, C_{0}\right]$ in Fig. 2.1, comprised of an unknown (or perhaps partially known) plant $P$ and a stabilizing controller $C_{0}$, is internally stable. Further suppose that based on the data collected from the stable closed-loop of $\left[P, C_{0}\right]$ the use of a new controller $C_{1}$ appears attractive-a typical aspect of recursive identification/controller redesign. How can one verify - without actual insertion in the closed-loop - if the introduction of the new controller $C_{1}$ will stabilize the plant and ensure a sensible level of closed-loop performance?

In the following two sections we will discuss techniques for verifying stability and performance with $C_{1}$ in advance of its insertion into the closed-loop using a limited 


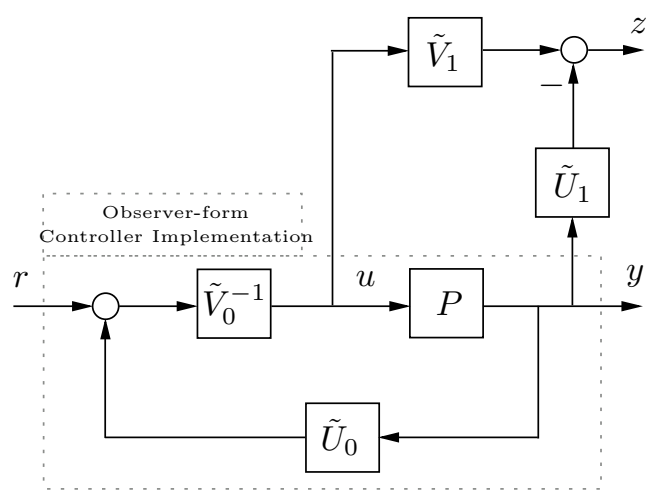

FIG. 4.1. Experimental setting: $C_{0}=\tilde{V}_{0}^{-1} \tilde{U}_{0}, C_{1}=\tilde{V}_{1}^{-1} \tilde{U}_{1}, T_{r \rightarrow z}: r \mapsto z$

amount of noisy input-output experimental data obtained from the stable closed-loop $\left[P, C_{0}\right]$. The verification tests will offer robustness to noise. First we shall present the stability verification results in the linear case.

4. Linear case: Stability Verification of $\left[P, C_{1}\right]$. The theorem below defines the experimental setting for the presentation of our stability verification tests.

Theorem 4.1. [9] Let $\left[P, C_{0}\right]$ be internally stable, $C_{0}=\tilde{V}_{0}^{-1} \tilde{U}_{0}$ and $C_{1}=\tilde{V}_{1}^{-1} \tilde{U}_{1}$ be left coprime factorizations over $\mathscr{R} \mathscr{H}_{\infty}$. Consider Fig. 4.1 and define $T_{r \rightarrow z}: r \mapsto z$ to be

$$
T_{r \rightarrow z}=\left[\begin{array}{ll}
-\tilde{U}_{1} & \tilde{V}_{1}
\end{array}\right]\left[\begin{array}{c}
P\left(I-C_{0} P\right)^{-1} \\
\left(I-C_{0} P\right)^{-1}
\end{array}\right] \tilde{V}_{0}^{-1}
$$

Then the following are equivalent:

a) $\left[P, C_{1}\right]$ is internally stable;

b) $\left(T_{r \rightarrow z}\right)^{-1} \in \mathscr{R} \mathscr{H}_{\infty}$;

c) $\operatorname{det} T_{r \rightarrow z}(j \omega) \neq 0 \forall \omega$ and $w n o \operatorname{det} T_{r \rightarrow z}=0$;

d) $\operatorname{det} T_{r \rightarrow z}(j \omega) \neq 0 \forall \omega$ and unwarg $\operatorname{det} T_{r \rightarrow z}(j \infty)=\operatorname{unwarg} \operatorname{det} T_{r \rightarrow z}(j 0)$.

Fig. 4.1 depicts the so-called "observer-form implementation" of controller $C_{0}$. Simple manipulation shows that the controller equation can also be rewritten as $u=$ $\left[\begin{array}{ll}-\tilde{U} & I+\tilde{V}\end{array}\right]\left[\begin{array}{l}y \\ u\end{array}\right]-r$ which justifies the reference to the observer-form configuration; see $[7,8]$ for a similar implementation.

Note that $P$ is unknown and hence one cannot explicitly construct $T_{r \rightarrow z}$ in closed form ${ }^{1}$. However, the stable mapping $T_{r \rightarrow z}: r \rightarrow z$ can be studied in a safe experiment,

\footnotetext{
${ }^{1}$ The results of Theorem 4.1 can be extended to include experimental settings involving alternative controller implementations. If $C_{0}$ is implemented only in the forward path, provided $C_{0}$ and $C_{1}$ are bi-proper and minimum-phase, one can study the stable mapping $T_{1}: r \rightarrow \tilde{r}$ with $\tilde{r}=C_{1}^{-1} u+y$, as a replacement for $T_{r \rightarrow z}$, in a safe experiment. Similarly, for the feedback-path implementation of $C_{0}$, if $C_{0}$ and $C_{1}$ have no right half-plane poles, the stable mapping $T_{2}: r \rightarrow \tilde{w}$ with $\tilde{w}=u+C_{1} y$ can be examined by utilizing the aformentioned results.
} 
i.e. one in which no instability can occur, as shown in Fig. 4.1. The reference signal $r$ and the constructed output signal $z$ (computed as a filtered version of the measured signals $\left[\begin{array}{l}y \\ u\end{array}\right]$ via $\tilde{K}_{1}$ ) can be used experimentally to infer the required properties of $T_{r \rightarrow z}$ for verifying condition (d) in Theorem 4.1. For the development of our data-based stability tests, the following assumptions are introduced.

Assumption 4.2. The factors $\tilde{V}_{0}$ and $\tilde{V}_{1}$ are such that $\tilde{V}_{0}(j \infty)=\tilde{V}_{1}(j \infty)=I$.

Assumption 4.3. The transfer functions $P C_{0}$ and $P C_{1}$ are strictly proper.

Notice that the transfer function $T_{r \rightarrow z}$ can be written as

$$
T_{r \rightarrow z}=\tilde{V}_{1}\left(I-C_{1} P\right)\left(I-C_{0} P\right)^{-1} \tilde{V}_{0}^{-1}
$$

for which under Assumptions 4.2 and 4.3 we have

$$
\operatorname{det} T_{r \rightarrow z}(j \infty)=\frac{\operatorname{det} \tilde{V}_{1}(j \infty)}{\operatorname{det} \tilde{V}_{0}(j \infty)} \frac{\operatorname{det}\left(I-C_{1} P\right)(j \infty)}{\operatorname{det}\left(I-C_{0} P\right)(j \infty)}=1
$$

Thus, $\operatorname{det} T_{r \rightarrow z}(j \infty)$ is strictly positive and known and will be used as a datum for the verification of condition (d) in Theorem 4.1. Next, note the following easy-to-use test.

TheOrem 4.4. [9] Let the suppositions of Theorem 4.1 and Assumptions 4.2 and 4.3 hold. Let $e_{i}$ denote a reference signal where a step is applied at the $i$-th input while the other inputs are kept at 0 . Perform $n$ experiments with reference signal $r(t)=e_{i}(t), i=1, \ldots, n$ and let $\bar{z}_{i}$ be the corresponding steady state output of $T_{r \rightarrow z}$ recorded in each experiment. Define $\bar{Z}=\left[\bar{z}_{1}, \ldots, \bar{z}_{n}\right]$. Then

$$
\left[P, C_{1}\right] \text { is internally stable } \Rightarrow \operatorname{det} \bar{Z}>0 .
$$

Thus if $\operatorname{det} \bar{Z} \leq 0$, stability of $\left[P, C_{1}\right]$ is falsified.

The experimental test in Theorem 4.4 is quite simple to carry out; it requires recording the steady state values of $m$ step responses. However such an experiment can only be used to check a necessary stability condition.

Condition (d) in Theorem 4.1 can be verified in both its necessary and sufficient parts by using more sophisticated identification techniques to compute the full frequency response for $T_{r \rightarrow z}$. However, this is not desirable on the grounds of complexity. In fact, one does not need to estimate the full frequency response of $T_{r \rightarrow z}$, but instead its frequency response up to a certain finite frequency $\omega_{0}$ will suffice. Moreover, experimental determination of a phase change known to be a multiple of $2 \pi$ allows room for some error in the measurements. A mechanism to estimate the frequency $\omega_{0}$ is advanced next by exploiting the structure of $T_{r \rightarrow z}$.

Lemma 4.5. [9] Let the suppositions of Theorem 4.1 hold. Then $T_{r \rightarrow z}$ can be 
expressed as

$$
\begin{aligned}
T_{r \rightarrow z} & =I+T^{\prime}, \\
T^{\prime} & =\left[\begin{array}{ll}
-\left(\tilde{U}_{1}-\tilde{U}_{0}\right) & \left(\tilde{V}_{1}-\tilde{V}_{0}\right)
\end{array}\right]\left[\begin{array}{c}
P\left(I-C_{0} P\right)^{-1} \\
\left(I-C_{0} P\right)^{-1}
\end{array}\right] \tilde{V}_{0}^{-1}
\end{aligned}
$$

The expression in (4.5) shows that $T_{r \rightarrow z}$ is the sum of a known term (i.e. $I$ ) and a term which, under Assumptions 4.2 and 4.3, is strictly proper. Thus measuring the frequency response of $T_{r \rightarrow z}$ up to a frequency, say $\omega_{0}$, where the response of $T^{\prime}$ has nearly vanished is enough to characterize the full frequency response of $T_{r \rightarrow z}$. This fact is utilized in Theorem 4.6.

TheOrem 4.6. [9] Suppose the hypotheses of Theorem 4.1 and Assumption 4.2 and 4.3 hold. Let $T^{\prime} \in \mathscr{R}_{\infty} \mathscr{H}_{\infty}^{n \times n}, T^{\prime}=T_{r \rightarrow z}-I$ as in (4.5), and $\omega_{0} \in[0, \infty)$ be a frequency such that

$$
\left\{\begin{array}{ll}
\rho\left(T^{\prime}(j \omega)\right)<1 & n=1 \\
\rho\left(T^{\prime}(j \omega)\right)<\sin \left(\frac{\pi}{n}\right) & n \geq 2
\end{array} \quad \forall \omega \geq \omega_{0}\right.
$$

Then the condition

$$
\left\{\begin{array}{l}
\operatorname{det} T_{r \rightarrow z}(j \omega) \neq 0 \forall \omega \in\left[0, \omega_{0}\right) \\
2 \pi \times \operatorname{nint}\left[\frac{\text { unwarg det } T_{r \rightarrow z}\left(j \omega_{0}\right)}{2 \pi}\right]=\text { unwarg } \operatorname{det} T_{r \rightarrow z}(j 0)
\end{array}\right.
$$

is equivalent to condition (d) in Theorem 4.1.

Note 4.7. Using the necessary and sufficient condition for the stability of $\left[P, C_{1}\right]$ in Theorem 4.6 requires estimation of the frequency response of the $T_{r \rightarrow z}(j \omega)$ up to $\omega_{0}$.

i. Recall that $\rho\left(T^{\prime}(j \omega)\right) \leq \bar{\sigma}\left(T^{\prime}(j \omega)\right)$ and under Assumptions 4.2 and 4.3, $\bar{\sigma}\left(T^{\prime}(j \omega)\right)$ tends to zero as $\omega$ tends to infinity. Practically, one may have a rough estimate of the bandwidth of $\left[P, C_{0}\right]$ which can be used to obtain an estimate of $\omega_{0}$ by assuming that $\bar{\sigma}\left(T^{\prime}(j \omega)\right)$ remains below the right-hand side of (4.6) over some known high-frequency region; a conservatively larger value makes the choice of $\omega_{0}$ robust.

ii. A small controller change certainly reduces the frequency $\omega_{0}$ and, as a consequence, reduces the experimental effort. The structure of $T^{\prime}$ in (4.5) is such that $\rho\left(T^{\prime}(j \omega)\right)$ depends on the size of the controller change; e.g. for the SISO case one can choose $\tilde{V}_{1}=\tilde{V}_{0}=1, \tilde{U}_{0}=C_{0}$ and $\tilde{U}_{1}=C_{1}$ resulting in $T^{\prime}=$ $\left(C_{0}-C_{1}\right) P /\left(1-C_{0} P\right)$. Techniques for finding an admissible size of the controller change are discussed in [12].

iii. The value $\operatorname{nint}\left[\operatorname{unwarg} \operatorname{det} T_{r \rightarrow z}\left(j \omega_{0}\right) / 2 \pi\right]$ is only used in condition (4.7); a rough estimate of unwarg $\operatorname{det} T_{r \rightarrow z}\left(j \omega_{0}\right) / 2 \pi$ is enough and hence the test can tolerate considerable estimation errors. 
iv. The estimate of the frequency response of $T_{r \rightarrow z}(j \omega)$ up to $\omega_{0}$ can be obtained via parametric [13] or non parametric [17] estimation methods. In practice, at each frequency one can use $\bar{\sigma}\left(T_{r \rightarrow z}\right) \leq\left\|T_{r \rightarrow z}\right\|_{F}=\left(\sum_{i, j}\left|T_{r \rightarrow z}^{i j}\right|^{2}\right)^{\frac{1}{2}}$ along with $\rho\left(T_{r \rightarrow z}(j \omega)\right) \leq \bar{\sigma}\left[T_{r \rightarrow z}(j \omega)\right]$ to find an upper bound on the eigenvalues of $T_{r \rightarrow z}(j \omega)$ in order to check (4.6). Alternatively the inequality $\bar{\sigma}\left[T_{r \rightarrow z}(j \omega)\right] \leq$ $\sqrt{n}\left\|T_{r \rightarrow z}(j \omega)\right\|_{1}$ can be utilized.

$v$. The unwrapped phase can be obtained by phase unwrapping techniques of [14].

5. Linear case: Performance Verification of $\left[P, C_{1}\right]$. We shall now build on the tools for projecting internal stability of $\left[P, C_{1}\right]$ presented in the previous section to enable measuring performance aspects of $\left[P, C_{1}\right]$ in advance. For this, recall that the plant $P$ is unknown, the controller $C_{0}=\tilde{V}_{0}^{-1} \tilde{U}_{0}$ is stabilizing and $C_{1}=\tilde{V}_{1}^{-1} \tilde{U}_{1}$ is verified to be stabilizing via the techniques of the previous section.

Lemma 5.1. Consider the interconnection in Fig. 4.1 and Definition 2.6. The mapping $T_{r \rightarrow z}: r \mapsto z$ in (4.1) and (4.2) can be expressed as

$$
T_{r \rightarrow z}=\left(\tilde{K}_{1} G\right)\left(\tilde{K}_{0} G\right)^{-1} .
$$

Proof.

$$
\begin{aligned}
T_{r \rightarrow z} & =\tilde{V}_{1}\left(I-C_{1} P\right)\left(I-C_{0} P\right)^{-1} \tilde{V}_{0}^{-1} \\
& =\left[\begin{array}{ll}
-\tilde{U}_{1} & \tilde{V}_{1}
\end{array}\right]\left[\begin{array}{c}
P\left(I-C_{0} P\right)^{-1} \\
\left(I-C_{0} P\right)^{-1}
\end{array}\right] \tilde{V}_{0}^{-1} \\
& =\tilde{K}_{1} \underbrace{\left[\begin{array}{c}
G\left(\tilde{K}_{0} G\right)^{-1}
\end{array}\right]}_{: r \rightarrow\left[\begin{array}{l}
y \\
u
\end{array}\right]} \\
& =\left(\tilde{K}_{1} G\right)\left(\tilde{K}_{0} G\right)^{-1}
\end{aligned}
$$

Lemma 5.2. Consider the interconnection in Fig. 4.1. Let $T_{r \rightarrow u}^{0}: r \mapsto u$ and $T_{r \rightarrow u}^{1}: r \mapsto u$ denote the reference-to-control transfer functions corresponding to the cases where controllers $C_{0}=\tilde{V}_{0}^{-1} \tilde{U}_{0}$ and $C_{1}=\tilde{V}_{1}^{-1} \tilde{U}_{1}$ were in the feedback loop, respectively. Then

$$
T_{r \rightarrow z}=\left(T_{r \rightarrow u}^{1}\right)^{-1} \quad T_{r \rightarrow u}^{0} .
$$

Proof. Note that $T_{r \rightarrow u}^{0}=\left(I-C_{0} P\right)^{-1} \tilde{V}_{0}$ and $T_{r \rightarrow u}^{1}=\left(I-C_{1} P\right)^{-1} \tilde{V}_{1}$. From (5.1),

$$
\begin{aligned}
T_{r \rightarrow z} & =\left(\tilde{K}_{1} G\right)\left(\tilde{K}_{0} G\right)^{-1} \\
& =\tilde{V}_{1}\left(I-C_{1} P\right)\left(I-C_{0} P\right)^{-1} \tilde{V}_{0}^{-1} \quad \text { via }(4.2) \\
& =\left(T_{r \rightarrow u}^{1}\right)^{-1} T_{r \rightarrow u}^{0}
\end{aligned}
$$


TheOREm 5.3. Suppose the hypotheses of Theorem 4.1 holds, and consider the setting in Fig. 4.1. If the conditions in Theorem 4.1 are satisfied,

$$
T_{r \rightarrow u}^{1}=T_{r \rightarrow u}^{0}\left(T_{r \rightarrow z}\right)^{-1} \in \mathscr{R} \mathscr{H}_{\infty} .
$$

Proof. Since $\left[P, C_{0}\right] \in \mathscr{R} \mathscr{H}_{\infty}$, we have $T_{r \rightarrow u}^{0} \in \mathscr{R} \mathscr{H}_{\infty}$. Condition b) in Theorem 4.1 ensures $\left(T_{r \rightarrow z}\right)^{-1} \in \mathscr{R} \mathscr{H}_{\infty}$ and thus $T_{r \rightarrow u}^{1} \in \mathscr{R} \mathscr{H}_{\infty}$.

Theorem 5.4. Consider the interconnection in Fig. 4.1. Let $T_{r \rightarrow y}^{0}: r \mapsto y$ and $T_{r \rightarrow y}^{1}: r \mapsto y$ denote the reference-to-output transfer functions corresponding to controllers $C_{0}=\tilde{V}_{0}^{-1} \tilde{U}_{0}$ and $C_{1}=\tilde{V}_{1}^{-1} \tilde{U}_{1}$, respectively. If $\left[P, C_{0}\right]$ and $\left[P, C_{1}\right]$ are internally stable,

$$
T_{r \rightarrow y}^{1}=T_{r \rightarrow y}^{0}\left(T_{r \rightarrow z}\right)^{-1} \in \mathscr{R} \mathscr{H}_{\infty} .
$$

Proof. The proof is easy via the arguments used to prove Theorem 5.3.

A connection between the transfer functions of the feedback setting in Fig. 2.1 and those of the interconnection in Fig. 4.1 can be established via calculating $H_{\left[P, C_{1}\right]}$. This is captured in the following theorem.

TheOrem 5.5. Consider the configurations in Fig. 2.1 and Fig. 4.1 and assume that $\left[P, C_{0}\right] \in \mathscr{R} \mathscr{H}_{\infty}$ and $\left[P, C_{1}\right] \in \mathscr{R} \mathscr{H}_{\infty}$. Then,

$$
H_{\left[P, C_{1}\right]}=\left[\begin{array}{c}
T_{r \rightarrow y}^{1} \\
T_{r \rightarrow u}^{1}
\end{array}\right]\left[\begin{array}{ll}
-\tilde{U}_{1} & \tilde{V}_{1}
\end{array}\right]=\left[\begin{array}{cc}
T_{r \rightarrow y}^{0} & \left(T_{r \rightarrow z}\right)^{-1} \\
T_{r \rightarrow u}^{0} & \left(T_{r \rightarrow z}\right)^{-1}
\end{array}\right]\left[\begin{array}{cc}
-\tilde{U}_{1} & \tilde{V}_{1}
\end{array}\right] \in \mathscr{R} \mathscr{H}_{\infty} .
$$

Proof. The proof is straightforward via the results in Theorems 5.3 and 5.4 and by noting the stable coprime factors of Definition 2.5 .

Corollary 5.6. If $\left\{\tilde{U}_{1}, \tilde{V}_{0}\right\}$ is a normalized lcf of the controller $C_{1}=\tilde{V}_{1}^{-1} \tilde{U}_{1}$, then

$$
\left\|H_{\left[P, C_{1}\right]}\right\|_{\infty}=\left\|\left[\begin{array}{ll}
T_{r \rightarrow y}^{0} & \left(T_{r \rightarrow z}\right)^{-1} \\
T_{r \rightarrow u}^{0} & \left(T_{r \rightarrow z}\right)^{-1}
\end{array}\right]\right\|_{\infty} .
$$

The aforementioned results, particularly in Theorem 5.3, can be utilized to verify the performance of $T_{r \rightarrow u}^{1}$ given the availability of performance aspects of $T_{r \rightarrow u}^{0}$ and $\left(T_{r \rightarrow z}\right)^{-1}$. Note that the closed-loop performance with the controller $C_{1}$ is determined by the size of the transfer functions in $H_{\left[P, C_{1}\right]}$, of which $T_{r \rightarrow u}^{1}$ is known to be important and is termed sensitivity function. Links to the classical and standard notions of performance are also presented in and around Definition 2.8 and Theorem 2.12. Next, we present results for projecting some performance aspects with $C_{1}$ prior to its actual insertion into the closed-loop, assuming that the transfer function $P$ is unknown.

Theorem 5.7. [6] Let $\left[P, C_{0}\right] \in \mathscr{R} \mathscr{H}_{\infty}$ and consider Fig. 4.1 and define $T_{r \rightarrow z}$ : $r \mapsto z$ to be as in (4.1). Let $b_{\left[P, C_{0}\right]}$ and $b_{\left[P, C_{1}\right]}$ be indices as in Definition 2.8. If 
$\left[P, C_{1}\right]$ is internally stable,

$$
b_{\left[P, C_{0}\right]}\left\|\left(T_{r \rightarrow z}\right)^{-1}\right\|_{\infty}^{-1} \leq b_{\left[P, C_{1}\right]} \leq b_{\left[P, C_{0}\right]}\left\|T_{r \rightarrow z}\right\|_{\infty} .
$$

The results in Theorem 5.7 is a flag indicating that the sensitivity of closed-loop $\left[P, C_{1}\right]$ might be very bad if $\left\|T_{r \rightarrow z}\right\|$ becomes very small. The pointwise version of Theorem 5.7 is given below.

Theorem 5.8 ( [6]). Suppose the hypothesis of Theorem 4.1 holds. Consider the setting in Fig. 4.1 and mapping $T_{r \rightarrow z}: r \mapsto z$ in (4.1). Then $\forall \omega$,

$$
\frac{1}{\bar{\sigma}\left[\left(T_{r \rightarrow z}(j \omega)\right)^{-1}\right]} \rho\left(P, C_{0}, \omega\right) \leq \rho\left(P, C_{1}, \omega\right) \leq \bar{\sigma}\left[^{-1}(j \omega)\right] \rho\left(P, C_{0}, \omega\right) .
$$

Theorem 5.9. Suppose $P$ is scalar in Fig. 4.1 and the hypothesis of Theorem 5.7 holds. Then,

$$
\left|T_{r \rightarrow z}(j \omega)\right| \rho\left(P, C_{0}, \omega\right)=\rho\left(P, C_{1}, \omega\right) \quad \forall \omega .
$$

Proof. From (5.1), we have

$$
\left(T_{r \rightarrow z} \tilde{K}_{0} G\right)(j \omega)=\left(\tilde{K}_{1} G\right)(j \omega) \quad \forall \omega
$$

which implies

$$
\underline{\sigma}\left[\left(T_{r \rightarrow z} \tilde{K}_{0} G\right)(j \omega)\right]=\underline{\sigma}\left[\left(\tilde{K}_{1} G\right)(j \omega)\right] \quad \forall \omega .
$$

Since $T_{r \rightarrow z}(j \omega)$ is a scalar here and $\left|T_{r \rightarrow z}(j \omega)\right|$ can be factored out, via (2.3) on obtains

$$
\left|T_{r \rightarrow z}(j \omega)\right| \rho\left(P, C_{0}\right)=\rho\left(P, C_{1}\right) .
$$

Corollary 5.10. Suppose P is scalar in Fig. 4.1 and the hypothesis of Theorem 5.7 hold. If $\left[P, C_{1}\right]$ is internally stable,

a. $G M_{\left[P, C_{1}\right]} \geq \frac{1+b_{\left[P, C_{0}\right]}\left\|\left(T_{r \rightarrow z}\right)^{-1}\right\|_{\infty}^{-1}}{1-b_{\left[P, C_{0}\right]}\left\|T_{r \rightarrow z}\right\|_{\infty}} ;$

b. $P M_{\left[P, C_{1}\right]} \geq 2 \arcsin \left(b_{\left[P, C_{0}\right]}\left\|\left(T_{r \rightarrow z}\right)^{-1}\right\|_{\infty}^{-1}\right)$.

Proof. The results follow from Theorems 2.12 and 5.7.

In our framework, $\left[P, C_{1}\right]$ is verified to be internally stable and

$$
T_{r \rightarrow u}^{1}(j \omega)=T_{r \rightarrow u}^{0}\left(T_{r \rightarrow z}\right)^{-1}(j \omega) .
$$

via Theorem 5.3. At each frequency, this relationship facilitates the computation of $\left|T_{r \rightarrow u}^{1}(j \omega)\right|$ and $\angle T_{r \rightarrow u}^{1}(j \omega)$. Note that verifying the performance of $\left[P, C_{1}\right]$ does not 
require the full frequency response of $T_{r \rightarrow u}^{1}(j \omega)$, but some frequency-domain characteristics discussed earlier to enable projection of the phase margin (PM) and gain margin (GM) of $\left[P, C_{1}\right]$, which also enables inferring other performance aspects. Other procedures can result in determining the bandwidth and other frequency-domain characteristics of $\left[P, C_{1}\right]$ via exploiting some properties of $T_{r \rightarrow u}^{1}$. One can also utilise the indirect measurement results in Lemma 5.7 and Corollary 5.10 to determine lower bounds on the gain margin (PM) and phase margin (GM) of $\left[P, C_{1}\right]$.

Next, an analysis framework is presented which enables the extension of the results in the linear case to the nonlinear case. We shall now assume that the 'unknown' plant and the controllers are all nonlinear.

6. Nonlinear case: Analysis. The problem of making a stability prediction becomes even harder when any combination of the plant and/or controllers are nonlinear. The scenario of switching from a linear to a nonlinear controller is also interesting and advocated when one seeks to improve some aspects of closed-loop performance without sacrificing some other aspects. For example, a nonlinear controller is used in place of a linear controller in [21] to achieve a faster rise-time without increasing the percentage overshoot.This is partly due to the fact that there exist fewer tools for analyzing nonlinear systems.

In this context, our results not only advance the preceding results for the linear plants and controllers, but also provide additional analysis tools for nonlinear systems. In particular, we shall extend the applicability of the kernel representation of a nonlinear system as a generalization for the existing results in the linear case for controller update in an adaptive control setting. As a generalization of left fractional descriptions of LTI systems, we consider kernel representations and shall review some notations and relevant definitions mostly borrowed from $[11,18]$ for ease of reference. Note that the results developed here are for classes of admissible nonlinear systems.

6.1. Kernel representations. Kernel representations generalize the ideas of left fractional description. Similarly, bounded kernel representations generalize the ideas of bounded left fractional descriptions. This is true for the coprime property. These properties are formally defined below.

Definition 6.1 (Kernel representation). Consider a causal operator $P: \mathscr{L}_{2 e}^{m} \rightarrow$ $\mathscr{L}_{2 e}^{k}$ with an initial condition space $\mathcal{X}_{P}$. Then a causal operator $R_{P}^{x_{P}}: \mathscr{L}_{2 e}^{m} \times \mathscr{L}_{2 e}^{k} \rightarrow$ $\mathscr{L}_{2 e}^{k}, \forall x_{P} \in \mathcal{X}_{P}$ is called a kernel representation of $P$ if $\forall x_{P} \in \mathcal{X}_{P}$ and $\forall u \in \mathscr{L}_{2 e}^{m}$, $y=P^{x_{P}} u \Leftrightarrow R_{P}^{x_{P}}(u, y)=0$ holds with $y \in \mathscr{L}_{2 e}^{k}$.

Definition 6.2 (Well-definedness). A kernel operator $R_{P}^{x_{P}}$ is well-defined if there exists the causal operator $\left(R_{P}^{x_{P}}\right)^{\#}: \mathscr{L}_{2 e}^{m} \times \mathscr{L}_{2 e}^{k} \rightarrow \mathscr{L}_{2 e}^{k}$ such that

$$
y=\left(R_{P}^{x_{P}}\right)^{\#}(u, z) \Leftrightarrow R_{P}^{x_{P}}(u, y)=z, \quad \forall u \in \mathscr{L}_{2 e}^{m}, y, z \in \mathscr{L}_{2 e}^{k}, \forall x_{P} \in \mathcal{X}_{P} .
$$

Here, all kernel representations are assumed to be well-defined. 


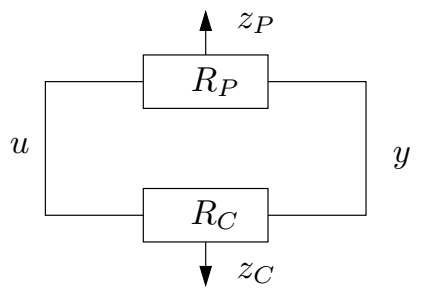

FIG. 6.1. Kernel representation of $[P, C]$ without any disturbance.

Definition 6.3 (Coprime). A bounded operator $R_{\Sigma}^{x_{P}}: \mathscr{L}_{2 e}^{m} \times \mathscr{L}_{2 e}^{k} \rightarrow \mathscr{L}_{2 e}^{k}$ is coprime if there exists a bounded operator $M^{x_{P}}: \mathscr{L}_{2 e}^{k} \rightarrow \mathscr{L}_{2 e}^{m} \times \mathscr{L}_{2 e}^{k}$ such that $R_{\Sigma}^{x_{P}} M^{x_{P}}=I \forall x_{P} \in \mathcal{X}_{\Sigma}$.

The analogous feedback configuration in Fig. 2.1 of $[P, C]$ in the kernel representation is shown in Fig. 6.1.

Definition 6.4 (Closed-loop kernel representation). Consider $P: \mathscr{L}_{2 e}^{m} \rightarrow$ $\mathscr{L}_{2 e}^{k}$ with $\mathcal{X}_{P}$ and $C: \mathscr{L}_{2 e}^{k} \rightarrow \mathscr{L}_{2 e}^{m}$ with $\mathcal{X}_{C}$, and kernel representations of $R_{P}$ : $\mathscr{L}_{2 e}^{m} \times \mathscr{L}_{2 e}^{k} \rightarrow \mathscr{L}_{2 e}^{k}$ and $R_{C}: \mathscr{L}_{2 e}^{k} \times \mathscr{L}_{2 e}^{m} \rightarrow \mathscr{L}_{2 e}^{m}$. If $R_{P}$ and $R_{C}$ are interconnected to form a feedback loop as shown in Fig. 6.1, then a closed-loop kernel representation $R_{[P, C]}: \mathscr{L}_{2 e}^{m} \times \mathscr{L}_{2 e}^{k} \rightarrow \mathscr{L}_{2 e}^{k} \times \mathscr{L}_{2 e}^{m}$ is defined as

$$
\left(z_{P}, z_{C}\right):=R_{[P, C]}^{\left(x_{p}, x_{c}\right)}(u, y)=\left(\begin{array}{l}
R_{P}(u, y) \\
R_{C}(y, u)
\end{array}\right) \quad \forall\left(x_{P}, x_{C}\right) \in \mathcal{X}_{P C},
$$

where $\mathcal{X}_{P C}:=\mathcal{X}_{P} \times \mathcal{X}_{C}$.

Note that the definition of the closed-loop kernel representation above is consistent with Definition 6.1, and the operator $R_{[P, C]}$ takes input $(u, y)$ and generates the output $\left(z_{P}, z_{C}\right)$ as arrows indicate in Fig. 6.1.

Next, we shall define the internal stability of systems in kernel representation, which is known as null stability. Note that if there is no external inputs in Fig. 2.1, i.e. $r_{1}, r_{2} \equiv 0$, then the feedback interconnection in Fig. 2.1 is equivalent to the kernel representation of Fig. 6.1; see [11] for further details.

Definition 6.5 (Null internally stability). $[P, C]$ with a weakly Lipschitz kernel representation $R_{[P, C]}:(u, y) \mapsto\left(z_{P}, z_{C}\right)$ of Fig. 6.1 is null well-posed if $\forall\left(x_{P}, x_{C}\right) \in$ $\mathcal{X}_{P C}, R_{[P, C]}^{-1}:\left(z_{P}, z_{C}\right) \mapsto(u, y)$ exists and it is weakly Lipschitz. Also, $[P, C]$ is null internally stable if it is null well-posed and $R_{[P, C]}^{-1}$ is bounded.

The null internal stability of the interconnection $[P, C]$ with $R_{[P, C]}$ is equivalent to the coprimeness of $R_{[P, C]}$; see [11].

7. New framework via kernel representation. Since left fractional descriptions are generally absent for nonlinear systems, we shall develop analysis tools and an experimental framework in the sequel utilising kernel representations.

Lemma 7.1. [2,4] Consider a closed-loop kernel operator $R_{[P, C]}$ as in (6.1) with $R_{P}: \mathscr{L}_{2 e}^{m} \times \mathscr{L}_{2 e}^{k} \rightarrow \mathscr{L}_{2 e}^{k}$ and $R_{C}: \mathscr{L}_{2 e}^{k} \times \mathscr{L}_{2 e}^{m} \rightarrow \mathscr{L}_{2 e}^{m}$ as shown in Fig. 6.1. Then 
$R_{[P, C]}$ is weakly Lipschitz (resp. bounded) if and only if $R_{P}$ and $R_{C}$ are both weakly Lipschitz (resp. bounded).

One can construct a mapping from $R_{\left[P, C_{0}\right]}$ to $R_{\left[P, C_{1}\right]}$, where $C_{0}$ and $C_{1}$ are two different controllers used with $P$.

Lemma 7.2. [2,4] Let $R_{P}, R_{C_{0}}$ and $R_{C_{1}}$ be bounded and weakly Lipschitz kernel representations for the operators $P, C_{0}$ and $C_{1}$, respectively. Suppose $R_{\left[P, C_{0}\right]}$ and $R_{\left[P, C_{1}\right]}$ are kernel representations of $\left[P, C_{0}\right]$ and $\left[P, C_{1}\right]$, respectively. Suppose $\left[P, C_{0}\right]$ is null internally stable; then one can define

$$
Q_{C_{0}}^{C_{1}}: \mathcal{Z}_{P C_{0}} \rightarrow \mathcal{Z}_{P C_{1}}:=R_{\left[P, C_{1}\right]}^{x_{P C_{1}}} \circ\left[R_{\left[P, C_{0}\right]}^{x_{P C_{0}}}\right]^{-1},
$$

where $Q_{C_{0}}^{C_{1}}\left(z_{P}, z_{C_{0}}\right)=\left(\left[Q_{C_{0}}^{C_{1}}\right]_{1}\left(z_{P}, z_{C_{0}}\right),\left[Q_{C_{0}}^{C_{1}}\right]_{2}\left(z_{P}, z_{C_{0}}\right)\right)$, and there holds

$$
\left[Q_{C_{0}}^{C_{1}}\right]_{1}\left(z_{P}, z_{C_{0}}\right)=z_{P} \in \mathcal{Z}_{P}
$$

Proof. For an external input $\left(z_{P}, z_{C_{0}}\right) \in \mathcal{Z}_{P C_{0}},\left[P, C_{0}\right]$ has the plant input $u$ and output $y$ related by

$$
R_{\left[P, C_{0}\right]}^{x_{P C_{0}}}(u, y)=\left(z_{P}, z_{C_{0}}\right)
$$

which includes $R_{P}^{x_{P}}(u, y)=z_{P}$. Since $\left[P, C_{0}\right]$ is null internally stable (ie. $\left[R_{\left[P, C_{0}\right]}^{x_{P C_{0}}}\right]^{-1}$ exists, is weakly Lipschitz and bounded), one has the inverse relationship of (7.2), $\left[R_{\left[P, C_{0}\right]}^{\left.x_{P C_{0}}\right]}\right]^{-1}\left(z_{P}, z_{C_{0}}\right)=(u, y)$, such that $R_{\left[P, C_{0}\right]}^{x_{P C_{0}}} \circ\left[R_{\left[P, C_{0}\right]}^{x_{P C_{0}}}\right]^{-1}=\left[R_{\left[P, C_{0}\right]}^{x_{P C_{0}}}\right]^{-1} \circ R_{\left[P, C_{0}\right]}^{x_{P C_{0}}}=I$. When $R_{P}^{x_{P}}(u, y)=z_{P}$ and $R_{C_{1}}^{x_{1}}(y, u)=z_{C_{1}}$ are interconnected, it becomes

$$
R_{\left[P, C_{1}\right]}^{x_{P C_{1}}}(u, y)=\left(z_{P}, z_{C_{1}}\right) .
$$

Hence, we can define $Q_{C_{0}}^{C_{1}}$ as shown in (7.1)

$$
\begin{aligned}
Q_{C_{0}}^{C_{1}}\left(z_{P}, z_{C_{0}}\right) & =R_{\left[P, C_{1}\right]}^{x_{P C_{1}}} \circ\left[R_{\left[P, C_{0}\right]}^{x_{P C_{0}}}\right]^{-1}\left(z_{P}, z_{C_{0}}\right) \\
& =R_{\left[P, C_{1}\right]}^{x_{P C_{1}}}(u, y)=\left(z_{P}, z_{C_{1}}\right) .
\end{aligned}
$$

Thus $\left[Q_{C_{0}}^{C_{1}}\right]_{1}\left(z_{P}, z_{C_{0}}\right)=z_{P}$.

Lemma 7.3. [2,4] The projection, proj: $\mathscr{L}_{2 e}^{m} \times \mathscr{L}_{2 e}^{k} \rightarrow \mathscr{L}_{2 e}^{m}$, defined as $\operatorname{proj}(a, b)=$ $a$, is weakly Lipschitz and bounded.

Now, we shall introduce an experimental setting for the nonlinear plant and the two controllers. Let $R_{P}$ and $R_{C_{0}}$ be the kernel representations of $P$ and $C_{0}$, respectively, where $R_{P}(u, y)=w$ and $R_{C_{0}}(y, u)=r$. If we assume that $\left[P, C_{0}\right]$ is null internally stable, we have a bounded operator $R_{\left[P, C_{0}\right]}^{-1}:(w, r) \mapsto(u, y)$. Then, one can attach $R_{C_{1}}:(y, u) \mapsto z$, the kernel operator of $C_{1}$, to $R_{\left[P, C_{0}\right]}^{-1}$ as shown in Fig. 7.1. Note that it terms of analogy between the feedback interconnection in the linear case, the external inputs are now labeled $(w, r)$ instead of $\left(r_{2}, r_{1}\right)$. 


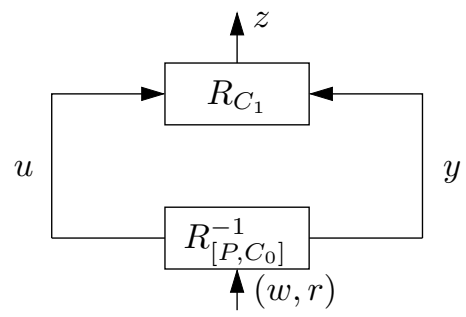

FIG. 7.1. Experiment setting for nonlinear case in Kernel representation.

THEOREM 7.4. Let $R_{P}, R_{C_{0}}$ and $R_{C_{1}}$ be bounded and weakly Lipschitz kernel representations for the operators $P, C_{0}$ and $C_{1}$, respectively. Suppose $R_{\left[P, C_{0}\right]}$ and $R_{\left[P, C_{1}\right]}$ are kernel representations of $\left[P, C_{0}\right]$ and $\left[P, C_{1}\right]$, respectively, and assume $\left[P, C_{0}\right]$ is null internally stable (ie. $\left[R_{\left[P, C_{0}\right]}^{\left.x_{P C_{0}}\right]}\right]^{-1}$ exists, is weakly Lipschitz and bounded). Then one can define a family of mappings $T_{w}: r \in \mathcal{Z}_{C_{0}} \mapsto z \in \mathcal{Z}_{C_{1}}$ parameterized by $w$

$$
T_{w}(r):=R_{C_{1}}^{x_{C_{1}}} \circ\left[R_{\left[P, C_{0}\right]}^{x_{P C_{0}}}\right]^{-1}(w, r)
$$

as shown in Fig. 7.1. Then the following are equivalent:

(a) $\left[P, C_{1}\right]$ is null internally stable;

(b) $T_{w}^{-1}: z \in \mathcal{Z}_{C_{1}} \mapsto r \in \mathcal{Z}_{C_{0}}$ exists, is weakly Lipschitz and parametrically bounded.

Proof.

Since $\left[P, C_{0}\right]$ is assumed to be null internally stable, Lemma 7.2 gives

$$
Q_{C_{0}}^{C_{1}}: \mathcal{Z}_{P C_{0}} \rightarrow \mathcal{Z}_{P C_{1}}:=R_{\left[P, C_{1}\right]}^{x_{P C_{1}}} \circ\left[R_{\left[P, C_{0}\right]}^{x_{P C_{0}}}\right]^{-1}
$$

where

$$
\left[Q_{C_{0}}^{C_{1}}\right]_{1}(w, r)=w
$$

One should note that

$$
T_{w}(r) \equiv\left[Q_{C_{0}}^{C_{1}}\right]_{2}(w, r) .
$$

$(\mathrm{a} \Rightarrow \mathrm{b})$ Now suppose $\left[P, C_{1}\right]$ is null internally stable (ie. by Definition 6.5 , $\left[R_{\left[P, C_{1}\right]}^{x_{P C_{1}}}\right]^{-1}$ exists, is weakly Lipschitz and bounded). By using Lemma 7.2 again, one can find

$$
Q_{C_{1}}^{C_{0}}: \mathcal{Z}_{P C_{1}} \rightarrow \mathcal{Z}_{P C_{0}}:=R_{\left[P, C_{0}\right]}^{x_{P C_{0}}} \circ\left[R_{\left[P, C_{1}\right]}^{x_{P C_{1}}}\right]^{-1}
$$

where

$$
\left[Q_{C_{1}}^{C_{0}}\right]_{1}(w, z)=w
$$


Note that $Q_{C_{1}}^{C_{0}}$ is, in fact, the inverse of $Q_{C_{0}}^{C_{1}}$. Now define

$$
S_{w}(z):=\left[Q_{C_{1}}^{C_{0}}\right]_{2}(w, z)
$$

and show that it is the inverse of $T_{w}$ for arbitrary but fixed $w$. First, observe that

$$
\begin{aligned}
& T_{w} \circ S_{w}(z)=T_{w} \circ\left[Q_{C_{1}}^{C_{0}}\right]_{2}(w, z) \text { via }(7.10) \\
& =\left[Q_{C_{0}}^{C_{1}}\right]_{2}\left(w,\left[Q_{C_{1}}^{C_{0}}\right]_{2}(w, z)\right) \text { via }(7.7) \\
& =\left[Q_{C_{0}}^{C_{1}}\right]_{2}\left(\left[Q_{C_{1}}^{C_{0}}\right]_{1}(w, z),\left[Q_{C_{1}}^{C_{0}}\right]_{2}(w, z)\right) \text { via }(7.9) \\
& =\left[Q_{C_{0}}^{C_{1}}\right]_{2} \circ Q_{C_{1}}^{C_{0}}(w, z)=R_{C_{1}}^{x_{C_{1}}} \circ\left[R_{\left[P, C_{1}\right.}^{x_{P C_{1}}}\right]^{-1}(w, z)=z .
\end{aligned}
$$

Second, note that

$$
\begin{aligned}
& S_{w} \circ T_{w}(r)=S_{w} \circ\left[Q_{C_{0}}^{C_{1}}\right]_{2}(w, r) \text { via }(7.7) \\
& =\left[Q_{C_{1}}^{C_{0}}\right]_{2}\left(w,\left[Q_{C_{0}}^{C_{1}}\right]_{2}(w, r)\right) \text { via }(7.10) \\
& =\left[Q_{C_{1}}^{C_{0}}\right]_{2}\left(\left[Q_{C_{0}}^{C_{1}}\right]_{1}(w, r),\left[Q_{C_{0}}^{C_{1}}\right]_{2}(w, r)\right) \text { via }(7.6) \\
& =\left[Q_{C_{1}}^{C_{0}}\right]_{2} \circ Q_{C_{0}}^{C_{1}}(w, r)=R_{C_{0}}^{x_{C_{0}}} \circ\left[R_{\left[P, C_{0}\right]}^{x_{P} C_{0}}\right]^{-1}(w, r)=r .
\end{aligned}
$$

Thus

$$
\begin{aligned}
T_{w}^{-1}(z) & :=S_{w}(z)=\left[Q_{C_{1}}^{C_{0}}\right]_{2}(w, z) \\
& =R_{C_{0}}^{x_{C_{0}}} \circ\left[R_{\left[P, C_{1}\right]}^{x_{P C_{1}}}\right]^{-1}(w, z)
\end{aligned}
$$

exists, and $T_{w}^{-1}$ is weakly Lipschitz since $R_{C_{0}}^{x_{C_{0}}}$ and $\left[R_{\left[P, C_{1}\right]}^{\left.x_{P C_{1}}\right]}\right]^{-1}$ are weakly Lipschitz, and the cascade is also weakly Lipschitz (see Remark 2.18). Furthermore, $T_{w}^{-1}$ is parametrically bounded since the cascade of two bounded operators is bounded.

$(\mathrm{a} \Leftarrow \mathrm{b})$ Suppose that for fixed $w$, there exists $T_{w}^{-1}: z \in \mathcal{Z}_{C_{1}} \mapsto r \in \mathcal{Z}_{C_{0}}$ such that

$$
T_{w} \circ T_{w}^{-1}=T_{w}^{-1} \circ T_{w}=I,
$$

with $T_{w}^{-1}$ weakly Lipschitz and parametrically bounded.

If we define

$$
W(w, z):=\left(w, T_{w}^{-1}(z)\right)=(w, r),
$$

then

$$
\begin{aligned}
W \circ Q_{C_{0}}^{C_{1}}(w, r) & =W\left(\left[Q_{C_{0}}^{C_{1}}\right]_{1}(w, r),\left[Q_{C_{0}}^{C_{1}}\right]_{2}(w, r)\right) \text { via }(7.5) \\
& =\left(\left[Q_{C_{0}}^{C_{1}}\right]_{1}(w, r), T_{w}^{-1} \circ\left[Q_{C_{0}}^{C_{1}}\right]_{2}(w, r)\right) \text { via }(7.15) \\
& =\left(w, T_{w}^{-1} \circ\left[Q_{C_{0}}^{C_{1}}\right]_{2}(w, r)\right) \text { via }(7.6) \\
& =\left(w, T_{w}^{-1} \circ T_{w}(r)\right)=(w, r) \quad \text { via }(7.14) .
\end{aligned}
$$


Similarly,

$$
\begin{aligned}
Q_{C_{0}}^{C_{1}} \circ W(w, z) & =Q_{C_{0}}^{C_{1}}(w, r) \text { via }(7.15) \\
& =\left(\left[Q_{C_{0}}^{C_{1}}\right]_{1}(w, r),\left[Q_{C_{0}}^{C_{1}}\right]_{2}(w, r)\right) \text { via }(7.5) \\
& =\left(w,\left[Q_{C_{0}}^{C_{1}}\right]_{2}(w, r)\right) \text { via }(7.6) \\
& =\left(w, T_{w}(r)\right)=(w, z) .
\end{aligned}
$$

Hence, $W$ is the inverse of $Q_{C_{0}}^{C_{1}}$ (ie. $W(w, z)=Q_{C_{1}}^{C_{0}}(w, z)$ ). This implies that $Q_{C_{1}}^{C_{0}}$ exists for all input $(w, z)$ and since

$$
Q_{C_{1}}^{C_{0}}: \mathcal{Z}_{P C_{1}} \rightarrow \mathcal{Z}_{P C_{0}}:=R_{\left[P, C_{0}\right]}^{x_{P C_{0}}} \circ\left[R_{\left[P, C_{1}\right]}^{x_{P C_{1}}}\right]^{-1},
$$

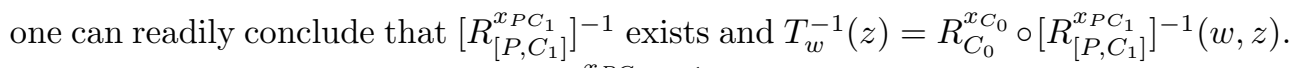
To finish off, we need to show that $\left[R_{\left[P, C_{1}\right]}^{\left.x_{P C_{1}}\right]^{-1}}\right.$ is weakly Lipschitz and bounded.

Observe first that $W_{1}(w, z)=w$ is a projection operator and by Lemma $7.3, W_{1}$ is weakly Lipschitz and bounded. Since $W_{2}(w, z)=T_{w}^{-1}(z)$ is weakly Lipschitz and parametrically bounded by hypothesis, $Q_{C_{1}}^{C_{0}}=W$ is weakly Lipschitz and bounded (as each component of $W(w, z)=\left(W_{1}(w, z), W_{2}(w, z)\right)$ is weakly Lipschitz and bounded).

Since $\left[P, C_{0}\right]$ is assumed to be null internally stable (ie. $\left[R_{\left[P, C_{0}\right]}^{\left.x_{P C_{0}}\right]}\right]^{-1}$ exists and weakly Lipschitz and bounded), one can conclude $\left[R_{\left[P, C_{1}\right]}^{x_{P C_{1}}}\right]^{-1}=\left[R_{\left[P, C_{0}\right]}^{x_{P C_{0}}}\right]^{-1} \circ Q_{C_{1}}^{C_{0}}$ is weakly Lipschitz (Remark 2.18) and bounded (as the cascade of two bounded operators is also bounded). Given Definition $6.5,\left[P, C_{1}\right]$ is null internally stable.

Note 7.5. In connection to the linear case with the plant $P$ and controllers $C_{0}=\tilde{V}_{0}^{-1} \tilde{U}_{0}$ and $C_{1}=\tilde{V}_{1}^{-1} \tilde{U}_{1}$, one has

$$
\begin{aligned}
y=P u & \Leftrightarrow \quad R_{P}^{x_{P}}(u, y)=0 \\
u=C_{0} y & \Leftrightarrow \quad R_{C_{0}}^{x_{C_{0}}}(y, u):=\left[\begin{array}{lll}
-\tilde{U}_{0} & \tilde{V}_{0}
\end{array}\right]\left(\begin{array}{l}
y \\
u
\end{array}\right)=0 .
\end{aligned}
$$

This implies that the interconnection of $\left[P, C_{0}\right]$ can be expressed with $(0, r) \in \mathcal{Z}_{P C_{0}}$ as

$$
(u, y)=\left[R_{\left[P, C_{0}\right]}^{\left.x_{P C_{0}}\right]}\right]^{-1}(0, r) \quad \Leftrightarrow \quad\left(\begin{array}{c}
y \\
u
\end{array}\right)=G\left(\tilde{K}_{0} G\right)^{-1} r
$$

Hence,

$$
T_{0}=R_{C_{1}}^{x_{C_{1}}} \circ\left[R_{\left[P, C_{0}\right]}^{x_{P}, x_{C_{0}}}\right]^{-1}=\left(\tilde{K}_{1} G\right)\left(\tilde{K}_{0} G\right)^{-1} .
$$

Indeed, (7.18) coincides with (5.1).

In order to show the connection between the results in the linear case and those of Theorem 7.4, the following theorem is presented next.

TheOREm 7.6. Suppose the hypotheses of Theorem 7.4 hold and consider the setting in Fig. 7.1. Assume that $P, C_{0}$ and $C_{1}$ are all LTI and define $z:=T_{w}(r)$ and $\tilde{z}:=T_{0}(r)$. Then $T_{0}^{-1}: r \mapsto \tilde{z}$ exists iff $T_{w}^{-1}: r \mapsto z$. 
Proof. Since $P, C_{0}$ and $C_{1}$ are all LTI, we have

$$
\begin{aligned}
T_{w}(r) & =R_{C_{1}}^{x_{C_{1}}} \circ\left[R_{\left[P, C_{0}\right]}^{\left.x_{P C_{0}}\right]}\right]^{-1}(w, r) \\
& =R_{C_{1}}^{x_{C_{1}}} \circ\left[R_{\left[P, C_{0}\right]}^{\left.x_{P C_{0}}\right]}\right]^{-1}(0, r)+R_{C_{1}} \circ\left[R_{\left[P, C_{0}\right]}\right]^{-1}(w, 0) \\
& =T_{0}(r)+T_{w}(0) .
\end{aligned}
$$

Note that the initial condition is considered on the term $T_{0}(r)$. One should also notice that for a fixed $w, T_{w}(0)$ can be regarded as a constant for all $r$.

$(\Rightarrow)$ Suppose $T_{0}^{-1}$ exists (ie. $T_{0}(r)=\tilde{z} \Leftrightarrow T_{0}^{-1}(\tilde{z})=r$ ). Since $z=\tilde{z}+T_{w}(0)$ (or $\left.\tilde{z}=z-T_{w}(0)\right)$, we have

$$
T_{0}(r)=z-T_{w}(0) \quad \Leftrightarrow \quad r=T_{0}^{-1}\left(z-T_{w}(0)\right)
$$

If we define $S_{w}(z):=T_{0}^{-1}\left(z-T_{w}(0)\right)$, then we have

$$
\begin{aligned}
& T_{w}\left(S_{w}(z)\right)=T_{w}\left(T_{0}^{-1}\left(z-T_{w}(0)\right)\right)=T_{w}(r)=z \\
& S_{w}\left(T_{w}(r)\right)=T_{0}^{-1}\left(z-T_{w}(0)\right)=T_{0}^{-1}(\tilde{z})=r .
\end{aligned}
$$

Hence $S_{w}$ is, in fact the inverse of $T_{w}$, that is

$$
T_{w}^{-1}(z):=T_{0}^{-1}\left(z-T_{w}(0)\right)
$$

$(\Leftarrow)$ If $T_{w}^{-1}$ exists for all $w$, then $T_{0}^{-1}$ also exists as it is a special case with $w=0$.

In principle, Theorem 7.4 offers a mechanism to verify the stability of the closedloop system $\left[P, C_{1}\right]$ by checking the invertibility of $T_{w}$, together with the weakly Lipschitz condition and boundedness of $T_{w}^{-1}$. One should note that as (7.4) indicates, $R_{P}$ (or at least a good approximation or model of $R_{P}$ ) is required in order to be able to compute $T_{w}$ or to check whether $T_{w}^{-1}$ exists. If $P$ was known, our Theorem 7.4 would provide a method to analyze $C_{1}$ before it is inserted into a stable closed-loop $\left[P, C_{0}\right]$. However, we want to verify whether the new $C_{1}$ will stabilize $P$ using only a limited data collected from $\left[P, C_{0}\right]$, where $P$ is unknown. This is explored in [4] and we have shortened the results here for the purpose of reducing complexity.

8. Conclusions. We have presented robust stability and performance validation tests for linear time-invariant systems which aim to project stability and some aspects of closed-loop performance with the introduction of a new controller $C_{1}$. The tests utilize a limited amount of experimental data obtained from the stable closed-loop interconnection $\left[P, C_{0}\right]$. One of the stability verification results of Section 4 uses step response properties of $T_{r \rightarrow z}$ in Fig. 4.1 to falsify the controller $C_{1}$, while Theorem 4.6 proposes a type of phase test analogous to the Nyquist criterion and utilized the noisy frequency response information of $T_{r \rightarrow z}$ up to a finite frequency $\omega_{0}$ to check if $C_{1}$ will 
stabilize the unknown plant $P$. It verifies that $C_{1}$ will be stabilizing (in place of $C_{0}$ ) if the Nyquist plot of $T_{r \rightarrow z}$ does not encircle the origin.

The performance verification results of Section 5 project performance of the closed-loop with $C_{1}$ before its insertion into the closed-loop and raise a red flag if, for example, at any frequencies $T_{r \rightarrow z}$ has small magnitude. Also, bounds on potential performance degradation have been developed.

The nonlinear extension of the results in the linear case has also been presented. Using the kernel representation, the analysis results of Section 6 has laid the foundation for the case when the plant and controllers are nonlinear and established connection to the linear case results.

Our current research focuses on extending the data-based tests of [4] to a larger class of controller structures. This is very vital in applying the results in the biomedical engineering areas and in particular for the development of control methodologies for drug dosing in hemodynamic (blood flow) management and control of consciousness.

\section{REFERENCES}

[1] B. D. O. Anderson and A. Dehghani, Challenges of adaptive control-past, permanent and future, Annual Reviews in Control, 32 (2008), pp. 123-135.

[2] S. H. Cha, A. Dehghani, And B. D. O. Anderson, Nonlinear analysis for verifying closed-loop stability before inserting a new controller, in Proceedings of the 10th European Control Conference, Budapest, August 2009, pp. 1095-1100.

[3] _ Safe nonlinear controller design with biomedical engineering applications, in Proceedings of the 7th Asian Control Conference, Hong Kong, August 2009, pp. 964-969.

[4] — - Verifying nonlinear controllers for stability utilizing closed-loop noisy data, in Proceedings of the 48th IEEE Conference on Decision and Control, Shanghai, December 2009, pp. $7657-7662$.

[5] A. Dehghani and B. D. O. Anderson, Stability and performance verification before switching in new controllers using closed-loop data, in Proceedings of the 49th IEEE Conference on Decision and Control, Atlanta, Georgia, USA, December 2010.

[6] A. Dehghani, B. D. O. Anderson, And S. H. Cha, Verifying closed-loop performance before inserting a new controller, in Proceedings of American Control Conference, Baltimore, June 2010.

[7] A. Dehghani, B. D. O. Anderson, And A. Lanzon, Unfalsified adaptive control: A new controller implementation and some remarks, in Proceedings of the 2007 European Control Conference, Kos, Greece, July 2007, pp. 709-716.

[8] A. Dehghani, A. Lanzon, and B. D. O. Anderson, A two degree of freedom $\mathscr{H}_{\infty}$ control design method for robust model matching, International Journal of Robust and Nonlinear Control, 16 (2006), pp. 467-483.

[9] A. Dehghani, A. Lecchini, A. Lanzon, and B. D. O. Anderson, Validating controllers for internal stability utilizing closed-loop data, IEEE Transactions on Automatic Control, 54 (2009), pp. $2719-2725$.

[10] S. Engell, T. Tometzki, and T. Wonghong, A new approach to adaptive unfalsified control, in Proceedings of the European Control Conference 2007, Kos, Greece, July 2007, pp. 13281333 . 
[11] K. Fujimoto, B. D. O. Anderson, And F. De Bruyne, A parameterization for closed-loop identification of nonlinear systems based on differentially coprime kernel representations, Automatica, 37 (2001), pp. 1893-1907.

[12] A. Lecchini, A. Lanzon, And B. D. O. Anderson, A model reference approach to safe controller changes in iterative identification and control, Automatica, 42 (2006), pp. 193-203.

[13] L. LJung, System Identification-Theory For the User, Prentice Hall, Upper Saddle River, NJ, second ed., 1999.

[14] R. MCGowan And R. Kuc, A direct relation between a signal time series and its unwrapped phase, IEEE Transactions on Acoustics, Speech, and Signal Processing, 30 (1982), pp. 719726.

[15] A. S. Monse, Supervisory control of families of linear set-point controllers part $i$. exact matching, IEEE Transactions on Automatic Control, 41 (1996), pp. 1413-1431.

[16] K. S. NARendra And J. Balakrishnan, Adaptive control using multiple models, IEEE Transactions on Automatic Control, 42 (1997), pp. 171-187.

[17] R. Pintelon and J. Schoukens, System Identification: a frequency domain approach, WileyIEEE Press, New York, 1979.

[18] A. J. VAN DeR Schaft, $\mathscr{L}_{2}$-gain and passivity techniques in nonlinear control, Springer Verlag, 2nd ed., 2000.

[19] M. VidYASAGAR, On the well-posedness of large-scale interconnected systems, IEEE Transactions on Automatic Control, 25 (1980), pp. 413-420.

[20] G. Vinnicombe, Uncertainty and Feedback: $\mathscr{H}_{\infty}$ loop-shaping and the $\nu$-gap metric, Imperial College Press, 2000.

[21] D. Williamson, Nonlinear compensation of linear processes, Automatica, 15 (1979), pp. 601608.

[22] K. Zhou And J. C. Doyle, Essentials of Robust Control, Prentice-Hall, 1998. 